\title{
Motivational intervention integrated into care of facial injury patients may reduce illicit drug use behaviours
}

\author{
Abstracted from \\ Shetty V, Murphy DA, Zigler C, Yamashita DD, Belin TR. \\ Randomized controlled trial of personalized motivational interventions in substance \\ using patients with facial injuries. / Oral Maxillofac Surg 2011; 69: 2396-2411. \\ Epub 2011 Apr 15. PubMed PMID: 21496991; PubMed Central \\ Address for correspondence: Dr Shetty, Oral and Maxillofacial Surgery, \\ University of California, Los Angeles, 10833 Le Conte Ave, Los Angeles, CA 90095, USA. \\ E-mail: vshetty@ucla.edu
}

\section{Question: In patients with a history of illicit drug and/or alcohol use and facial injuries requiring follow up does personalised motivational intervention (PMI) provided during the period reduce risky behaviours?}

\section{Design Randomised controlled trial}

Intervention All participants attended two counselling sessions: the first occurred shortly after study entry and the second occurred four to six weeks later. Sessions were provided by a trained practitioner in English or Spanish, participants in the control group received brief health information, subjects in the PMI group received an individualised behavioural intervention. Participants were followed up at six and 12 months.

Outcome measure Primary outcomes included changes in patterns of illicit drug use measured using the Texas Christian University Drug Screen (TCUDS) and the number of drug-using days, for all drugs as well as for the primary drug of choice, and changes in patterns of alcohol use measured with the Alcohol Use Disorders Identification Test (AUDIT), days of alcohol use and use of alcohol to intoxication. Results Two hundred and eighteen were randomised into the intervention $(n=118)$ and control $(n=100)$ groups. $51.7 \%$ of the PMI group and $62.0 \%$ in the control group completed the six-month follow-up, and $43.2 \%$ of the intervention group and $57.0 \%$ of controls completed the 12-month follow-up. Those in the PMI group showed statistically significant declines in drug use at both the six- and 12-month assessments. The effect on lowering illicit drug use was greatest at the six-month assessment but had weakened by the oneyear follow-up. The efficacy of the PMI was moderated by the severity of an individual's initial drug use, with individuals with greater drug use dependency at baseline seen to have larger intervention effects, as did individuals who were most aware of their drug problem and willing to change their substance use behaviours. Changes in alcohol use did not differ significantly between the intervention and control groups, irrespective of an individuals' recognition of the alcohol problem or willingness to take steps to address it.

Conclusions A culturally competent, motivational intervention integrated into the care of vulnerable patients with facial injury can reduce illicit drug use behaviours. Subgroups of injured patients appear to benefit most from such personalised motivational interventions. A better articulation of target populations, intervention content and delivery would allow for directed interventions and an appropriate focusing of limited time and health care resources.

\section{Commentary}

This randomised controlled trial aimed to test the effectiveness of personalised motivational interventions to reduce illicit drug use or alcohol consumption behaviour when appended to standard facial injury care in a trauma setting.

The association between alcohol and drug misuse and oro-facial injury through interpersonal violence is such that it has been recommended that all trauma admissions be considered an expression of underlying substance misuse and trigger screening and behavioural interventions to address substance misuse behaviours. Research indicates that alcohol screening and brief interventions are effective and cost effective in reducing alcohol consumption. ${ }^{1,2}$ Combined with the 'teachable moment' or post-injury period of heightened receptivity this environment presents an opportunity to contextualise the substance misuse problem and potentially enhance the outcomes.

This paper begins with a summary of the, often violent, association between alcohol and illicit drug misuse, and highlights the potential role of trauma staff.

The methodology appears appropriate for a randomised controlled trial and is described as a two-arm computer randomisation with blinded allocation. The participants were randomised into either the Personalised Motivational Intervention (PMI) or Health Information (HI) control groups to receive basic advice or an intervention following a scripted pattern (to maintain fidelity and ensure consistency of themes) lasting 15 to 60 minutes. Aspects of the methodological write-up lacked clarity. The HI group intervention was described in just two lines reporting the delivery of nonspecific brief health information, while the PMI group received in-depth explanation. Although both groups reportedly received two sessions there is no mention or explanation of a $2^{\text {nd }}$ reinforcement session for HI. This may explain, as the authors report, the equivocal findings, no greater effect from a more intensive intervention, indeed greater declines in harmful drinking in the HI group. The HI group intervention appears successful but the exact process is unclear.

The primary assessment instrument for drug misuse was the Texas Christian University Drug Screen (TCUDS), which seems to have good test-retest validity and overall accuracy. For alcohol misuse the Alcohol Use Disorders Identification Tool (AUDIT) has high specificity and sensitivity for alcohol misuse with a threshold for alcohol misuse of $\geq 8$.

The PMI group showed statistically significant declines in their TCUDS scores at both six and 12 month assessment, with 
HI showing a lesser effect. Drug-using days at the six month assessment had fallen, but decayed back to baseline pattern in both groups.

No significant differences in alcohol measure were found between groups at any of the assessment time points. All participants who screened positive for alcohol misuse, irrespective of group assignment, had decreases in AUDIT scores at six and 12 months. Contrary to expectation, the individualised interventions did not appear to influence alcohol use any more than did simple information only.

The success of alcohol moderation interventions has seen a campaign to expand their use in wider settings such as maxillofacial trauma units, A\&E and dentistry. One known barrier to introducing these measures is the belief that their delivery needs to be complex and time consuming. One conclusion from this paper is that alcohol advice in the form of basic information (HI) is as effective at reducing AUDIT scores compared with longer and more complex interventions. In contrast, interventions for drug misuse may require 'booster interventions' following effect decay suggesting the role of the maxillofacial trauma team may be more cost effective in identification and signposting to specialist drug services rather than developing complex personal interventions.

Tackling alcohol misuse in facial trauma patients is important because alcohol is often influential in the causation of injury, and maxillofacial trauma is a recurrent phenomenon in some patients. ${ }^{3}$ The introduction of simple alcohol advice interventions in maxillofacial trauma units may prove to be both realistic and cost-effective in reducing alcohol consumption patterns in this patient group.

\section{Simon Shepherd}

University of Dundee, Dental School, Park Place, Dundee, Scotland.

1. Scottish Intercollegiate Guidelines Network. The management of harmful drinking and alcohol dependence in primary care : a national clinical guideline. Edinburgh: Scottish Intercollegiate Guidelines Network; 2003.

2. Kaner $\mathrm{EF}$, Beyer $\mathrm{F}$, Dickinson $\mathrm{HO}$, et al. Effectiveness of brief alcohol interventions in primary care populations. Cochrane Database Syst Rev. 2007 (2): CD004148.

3. Goodall CA, Ayoub AF, Crawford A, et al. Nurse-delivered brief interventions for hazardous drinkers with alcohol-related facial trauma: a prospective randomised controlled trial. Br J Oral Maxillofac Surg 2008; 46: 96-101.

Evidence-Based Dentistry (2012) 13, 87-88. doi:10.1038/sj.ebd.6400880 